\title{
44609 - MUTATIONS IN RYR1 GENE ASSOCIATED WITH MALIGNANT HYPERTHERMIA AND A NON-ANAESTHETIC PHENOTYPE
}

\section{Julian Loke, Malignant Hyperthermia Investigation Unit, University of Toronto, ON, Canada; \\ Natasha Kraeva, Malignant Hyperthermia Investigation Unit, University of Toronto; \\ David H MacLennan, Banting and Best Dept of Medical Research, University of \\ Toronto;}

PURPOSE: To report a genotype-phenotype association between the presence of two novel mutations in the Calcium Release Channel gene, RYR1, in a patient with a history of both anaesthetic related malignant hyperthermia and frequent episodes of muscular rigidity in the absence of any anaesthetic.

CLINICAL FEATURES: An 18 year old patient was referred for assessment with a past history of masseter muscle rigidity (MMR) during inguinal herniorraphy at the age of 6 years. Postoperative Creatine Kinase (CK) rose to 5362 IU/L, but there was no associated fever, metabolic nor respiratory acidosis. At that time, the patient had not reached minimum weight criteria for muscle biopsy testing.

At the age of 15 years the patient experienced episodes of generalized body rigidity, producing dyspnoea associated with chest rigidity, and painful calf spasms. Up to 100 episodes were reported by the patient to have occurred over a 3 year period, and though not associated with any anaesthetic, were apparently associated with hot weather and emotional stress, such as awaiting injection for vaccination. Resting CK levels were normal. EMG and EEG examinations were unable to distinguish between seizures and psuedoseizures, so the patient was placed on antiepileptic therapy (valproate and lamotrigene) by a specialist neurologist.

At the age of 18 years, the patient submitted to skeletal muscle biopsy. Caffeine-halothane contracture testing (CHCT) showed a 2.6 gram weight contracture response to $2 \mathrm{mM}$ caffeine [Normal $<0.3 \mathrm{gm} \mathrm{wt}$ ]; and 6.8 gram weight contracture response to $3 \%$ halothane [Normal $<0.5$ gm wt]. The CHCT result was interpreted as Malignant Hyperthermia Susceptibility. Histopathology confirmed normal skeletal muscle architecture, and failed to demonstrate central cores.

Following institutional REB approval and individual informed consent for genetic study and scientific publication, DNA and RNA were isolated from muscle and blood samples of the patient and RYR1 transcript was analyzed following an established method.1 Sequencing of the complete 15 kilobase RYR1 transcript revealed the presence of two novel mutations: $625 \mathrm{G}>\mathrm{A}$ in exon 7 corresponding to a mutation from glutamic acid at a position 209 to lysine (E209K), and another mutation $7007 \mathrm{G}>\mathrm{A}$ corresponding to a mutation of arginine at position 2336 to histidine $(\mathrm{R} 2336 \mathrm{H})$. Applying in silico SIFT analysis, 2 the E209K mutation is predicted to be benign, while the $\mathrm{R} 2336 \mathrm{H}$ mutations is predicted to deleterious. Genetic pedigree analysis revealed that they are syntenic. Functional expression testing is required to confirm this prediction.

CONCLUSION: This is the first reported association between two novel mutations, one in exon 7 and another in exon 43, of the RYR1 gene that is associated with both an 
anaesthetic and a non-anaesthetic phenotype.

REFERENCES:

1. Anesthesiol 2003 99:289-296

2. Nucleic Acids Res 2003 31:3812-3814 